\title{
A GLRT FOR RADAR DETECTION IN THE PRESENCE OF COMPOUND-GAUSSIAN CLUTTER AND ADDITIVE WHITE GAUSSIAN NOISE
}

\author{
Bin Liu, Biao Chen \\ Syracuse University \\ Dept of EECS, Syracuse, NY 13244 \\ email : biliu\{bichen\}@ecs.syr.edu
}

\author{
James H. Michels
}

\author{
AFRL/SNRT \\ 26 Electronic Pkwy, Rome, NY 13441 \\ email: james.michels@rl.af.mil
}

\begin{abstract}
This paper addresses the detection problem for a signal with constant, but unknown amplitude in disturbance containing compound-Gaussian clutter plus additive white noise. Specifically, a new generalized likelihood ratio test (GLRT) is presented for the clairvoyant case. Previously developed GLRT expressions addressing the compound-Gaussian clutter problem have considered the 'clutter only' case assuming negligible additive white noise power. Performance evaluation is considered in the context of space-time adaptive processing (STAP) for an airborne radar application. We show improved performance of the new GLRT over previously developed tests.
\end{abstract}

\section{INTRODUCTION}

Space-time adaptive processing (STAP) has had a significant impact on advanced airborne AMTI and GMTI radar systems. Joint processing of spatialtemporal observations significantly improves the output signal-to-interference plus noise (SINR) power ratio that in turn provides improved detection performance.

Within the last decade, there has been considerable interest in the STAP detection problem in the presence of compound-Gaussian clutter $[1,2,3$, $4,5,6]$. For this clutter model, the clutter vector $\mathbf{c}$ is expressed as $\mathbf{c}=\sqrt{s} \mathbf{g}$ where $\mathbf{g}$ is complex Gaussian with covariance matrix $\boldsymbol{\Sigma}$ and $s$ is a non-negative scalar, unknown random clutter component (also called texture component) statistically independent of $\mathbf{g}$. In $[1,2]$, the multichannel radar detection problem was formulated as a binary detection problem; i.e., given complex observation data vector $\mathbf{x}$ where $\mathbf{x} \in C^{J N}$ ( $J$ channels, $N$ pulses), we

This work was supported by AFRL under Cooperative Agreement F30602-01-2-0525. choose between the following hypotheses

$$
\begin{array}{ll}
\mathbf{H}_{0} & \mathbf{x}=c=\sqrt{s} \mathbf{g} \\
\mathbf{H}_{1} & \mathbf{x}=a \mathbf{v}+\mathbf{c}=a \mathbf{v}+\sqrt{s} \mathbf{g}
\end{array}
$$

where $\mathbf{v}$ is the steering vector and $a$ is the unknown signal amplitude. It is worth mentioning that a widely referenced class of random processes, the socalled spherically invariant random process (SIRP) is a special class of compound-Gaussian by imposing a parametric model on the scalar (spiky) term which is assumed to remain constant for a coherrent processing interval (CPI). Examples for SIRP clutter $[7,8]$ include the $\mathrm{K}$ distribution and Weibull distribution for specific shape parameter values.

A generalized likelihood ratio test (GLRT) for the detection problem in (1) was developed in [2] which resulted in the following test statistic

$$
\Gamma_{1}=\frac{\left|\mathbf{x}^{H} \boldsymbol{\Sigma}^{-1} \mathbf{v}\right|^{2}}{\left(\mathbf{x}^{H} \boldsymbol{\Sigma}^{-1} \mathbf{x}\right)\left(\mathbf{v}^{H} \boldsymbol{\Sigma}^{-1} \mathbf{v}\right)}
$$

where $\boldsymbol{\Sigma}=E\left[\mathrm{gg}^{H}\right]$. This statistic is herein termed as the normalized matched filter (NMF) due to the fact that it contains a normalizing data dependent quadratic term to the well-known matched filter detector for Gaussian disturbances. We note that the same statistic was derived in [1] as an asymptotic optimum test for compound-Gaussian disturbance modeled as SIRP. The performance of adaptive versions of (2), known as the NAMF, was presented in [4].

Alternatively, an adaptive test called the adaptive coherence/cosine estimator (ACE) was developed for a Gaussian disturbance model with a scale change between test and training data and shown to be invariant $[9,10,11,12]$. Specifically, for a Gaussian disturbance vector $\mathbf{d}$ with covariance ma$\operatorname{trix} \mathbf{R}_{\mathbf{d}}, \mathbf{x} \sim C N\left(a \mathbf{v}, \eta^{2} \mathbf{R}_{\mathbf{d}}\right)$ under $\mathbf{H}_{1}$ and $\mathbf{x} \sim$ $C N\left(\mathbf{0}, \eta^{2} \mathbf{R}_{\mathbf{d}}\right)$ under $\mathbf{H}_{0}$, while the training data $\mathbf{z}_{k} \sim$ $C N\left(\mathbf{0}, \mathbf{R}_{\mathbf{d}}\right)$ for $k=1, \cdots, K$. Thus the variances of 
the training and test data differ by the factor $\eta^{2}$. For this model, the form of the ACE test is identical to (2) except that $\boldsymbol{\Sigma}$ is replaced by the maximum likelihood (ML) estimate; i.e., the sample covariance matrix $\hat{\mathbf{R}}_{\mathbf{d}} \triangleq \frac{1}{K} \sum_{k} \mathbf{z}_{k} \mathbf{z}_{k}^{H}$. The ACE test was noted to be CFAR for Gaussian processes with respect to scale change, a direct result of its invariance property. For the problem involving the compoundGaussian clutter modeled as an SIRP with power or scale changes over all range cells, $\hat{\mathbf{R}}_{\mathbf{d}}$ is no longer the ML estimator and no optimality claims for the ACE test can be made $[13,14]$.

In this paper, we consider the more general binary detection problem in which the observation data vector $\mathbf{x}$ also contains additive white noise. We show through numerical examples that the NMF derived using the clutter-only model suffers significant performance loss in terms of the CFAR property and detection probability in the presence of additive white Gaussian noise. Motivated by this, we develop a generalized likelihood ratio test (GLRT) for this problem.

The paper is organized as follows. In the next Section, the detection problem with both compound Gaussian and white Gaussian disturbance is presented and a generalized likelihood ratio test is developed. In Section III, we compare the detection performance of the new GLRT method with the NMF statistic along with some discussion on the CFAR property. We conclude in Section IV.

\section{A GLRT FOR DETECTION WITH BOTH COMPOUND-GAUSSIAN CLUTTER AND GAUSSIAN NOISE}

The detection problem with disturbance containing compound-Gaussian clutter and additive white Gaussian noise can be posed as the following hypotheses test:

$$
\begin{array}{ll}
\mathbf{H}_{0} & \mathbf{x}=\mathbf{c}+\mathbf{n} \\
\mathbf{H}_{1} & \mathbf{x}=a \mathbf{v}+\mathbf{c}+\mathbf{n}
\end{array}
$$

where $\mathbf{n} \sim \mathcal{C} \mathcal{N}\left(0, \sigma^{2} \mathbf{I}\right)$; i.e., $\mathbf{n}$ is complex white Gaussian noise assumed independent of the clutter $\mathbf{c}=$ $\sqrt{s}$ g. Therefore the disturbance covariance matrix is $\mathbf{M}=s \boldsymbol{\Sigma}+\sigma^{2} \mathbf{I}$. For this problem, the NAMF and ACE tests also lose the CFAR feature as reported in $[13,14]$.

For the detection problem specified in (3), a GLRT can be written straightforwardly as

$$
\begin{aligned}
\Gamma_{2} & =\frac{\max _{a, s, \sigma^{2}} f\left(\mathbf{x} \mid a, s, \sigma^{2} ; \mathbf{H}_{1}\right)}{\max _{s, \sigma^{2}} f\left(\mathbf{x} \mid s, \sigma^{2} ; \mathbf{H}_{0}\right)} \\
& =\frac{\max _{a, s, \sigma^{2}} \frac{1}{\|\mathbf{M}\|} \exp \left[-(\mathbf{x}-a \mathbf{v})^{H} \mathbf{M}^{-1}(\mathbf{x}-a \mathbf{v})\right]}{\max _{s, \sigma^{2}} \frac{1}{\|\mathbf{M}\|} \exp \left[-\mathbf{x}^{H} \mathbf{M}^{-1} \mathbf{x}\right]}
\end{aligned}
$$

Thus, we must now solve for the maximum likelihood estimates of all parameters under the two hypotheses. This is addressed next.

For STAP applications with compound-Gaussian clutter, while the clutter texture power at the test cell may be unknown, the additive white Gaussian noise power may be available from the operational system. We therefore distinguish the following two cases: (1) $\sigma^{2}$ known and (2) $\sigma^{2}$ unknown. As in [2], we assume that the clutter covariance structure, $\boldsymbol{\Sigma}$, is known.

\subsection{Known $\sigma^{2}$}

For the known noise power case, we assume without loss of generality that $\sigma^{2}=1$ as the observations can always be properly normalized. Therefore we need to solve the ML equations for $s$ and $a$ under the $\mathbf{H}_{1}$ hypothesis and for $s$ under the $\mathbf{H}_{0}$ hypothesis. The estimators are developed in Appendix A. They amount to solving a set of nonlinear equations that are summarized below.

- $\mathbf{H}_{1}$ hypothesis:

$$
\begin{aligned}
\operatorname{Tr}\left(\mathbf{\Sigma} \mathbf{M}^{-1}\right) & =(\mathbf{x}-a \mathbf{v})^{H} \mathbf{\Sigma} \mathbf{M}^{-2}(\mathbf{x}-a \mathbf{v}) \\
a & =\frac{\mathbf{v}^{H} \mathbf{M}^{-1} \mathbf{x}}{\mathbf{v}^{H} \mathbf{M}^{-1} \mathbf{v}}
\end{aligned}
$$

- $\mathbf{H}_{0}$ hypothesis:

$$
\operatorname{Tr}\left(\mathbf{\Sigma} \mathbf{M}^{-1}\right)=\mathbf{x}^{H} \mathbf{\Sigma} \mathbf{M}^{-2} \mathbf{x}
$$

\subsection{Unknown $\sigma^{2}$}

If $\sigma^{2}$ is unknown, we also need to find its ML estimate under $\mathbf{H}_{0}$ and $\mathbf{H}_{1}$. The estimates for $s$ and $a$ remain the same as noted above. The following $\mathrm{ML}$ equations result for the estimate of $\sigma^{2}$ :

- $\mathbf{H}_{1}$ hypothesis:

$$
\operatorname{Tr}\left(\mathbf{M}^{-1}\right)=(\mathbf{x}-a \mathbf{v})^{H} \mathbf{M}^{-2}(\mathbf{x}-a \mathbf{v})
$$

- $\mathbf{H}_{0}$ hypothesis:

$$
\operatorname{Tr}\left(\mathbf{M}^{-1}\right)=\mathbf{x}^{H} \mathbf{M}^{-2} \mathbf{x}
$$

It can be shown that the solutions of these equations are unique under the assumption that clutter to noise power is large. Then numerical procedures can be constructed easily to solve the above equations to obtain the ML estimates. These ML estimates are then used in (4) to obtain the GLRT statistic for detection. 


\section{SIMULATION RESULTS}

\subsection{Performance comparison}

In this section, we use numerical examples to compare the proposed GLRT method with the NMF developed in $[1,2]$. In the first example, we use two channels, four pulses, and the average clutter/noise power ratio $C N R=40 d B$. The output signal to interference and noise power ratio (SINR), defined as $10 \log _{10}|a|^{2} \mathbf{v}^{H} \mathbf{M}^{-1} \mathbf{v}$, is fixed at $6 \mathrm{~dB}$. The clutter assumes a $\mathrm{K}$ distribution with a shape parameter $\alpha=0.1$. The clutter ridge lies along the diagonal in the normalized Doppler-spatial frequency domain. The target signal is located at $0^{\circ}$ azimuth and 0.15 normalized Doppler frequency in the spatialtemporal (Doppler) domain and the clutter has one lag tempral correlation $u_{t}=0.999$.

Fig. 1 gives the ROC curves of the two statistics, namely the NMF and the proposed GLRT statistic. For the cases of both known $\sigma^{2}$ and unknown $\sigma^{2}$, the proposed GLRT of (4) outperforms the NMF of (2) by a significant margin. In the second example, we use a two channel thirty two pulse example which is otherwise identical to the previous case. Clearly from Fig. 2, a similar performance advantage of the GLRT is observed. The only difference is that the GLRT with known $\sigma^{2}$ and unknown $\sigma^{2}$ have almost identical performance for this higher dimensional example. This is due to the improved estimation performance for $\sigma^{2}$ resulting from the increased data size in this higher dimension case.

Fig. 3 gives the probability of detection versus SINR for a fixed probability of false alarm at $10^{-3}$. Clearly, the proposed GLRT outperforms NMF, especially in the low SINR region.

\subsection{Dicussion on the CFAR property}

In the absence of white Gaussian noise, the NMF of (2) has the desired CFAR property, i.e., the false alarm rate is independent of the covariance structure $\boldsymbol{\Sigma}$ and the clutter power term $s$. In the context of $\mathrm{K}$ distributed clutter, the CFAR with respect to power variation implies that it is CFAR with respect to the shape parameter. In Fig. 4, the probability of false alarm as a function of the shape parameter is obtained via simulation for a threshold chosen such that the nominal false alarm rate is $10^{-3}$ in the clutter-only case. The average CNR is again fixed at $40 d B$. Clearly, the probability of false alarm changes significantly as a function of the shape parameter in the presence of additive noise.

In Fig. 5, using the same parameters as in the first example, the probability of false alarm of the proposed GLRT is given for a fixed threshold for the $\sigma^{2}$ known case. Notice that if the clutter texture term $s$ is perfectly known, then the problem specified in (3) is a simple Gaussian clutter with known covariance matrix $\mathbf{M}$ and the detection statistic in (4) is reduced to the matched filter for Gaussian disturbance. Hence, it is clearly CFAR with respect to $s$. The fact that we have to estimate $s$ changes the CFAR property as shown in Fig. 5, most noticeably in the region with very small shape parameter. In this particular example, we notice that the proposed GLRT is still CFAR with respect to the shape parameter when it is greater than 0.1. The reason is as follows. The ML estimate of $s$ is likely to be very accurate for large CNR. However, at very low shape parameter, the variance of the clutter texture term $s$ becomes large. Therefore, even if the average CNR is kept at $40 d B$, the likelihood of having smaller CNR increases. This results in a larger error variance of estimate of $s$ which in turn affects the CFAR property of the proposed statistic. Further analysis with respect to the CFAR property will be performed in the future.

\section{CONCLUSIONS}

In this paper, we consider the detection problem for the case of unknown, constant signal amplitude in the presence of compound-Gaussian clutter plus additive white noise. Here, we address the case of known covariance for the Gaussian clutter component (speckle component), but unknown texture parameter. A GLRT method is derived for this problem and shown to outperform the NMF developed for the clutter-only case, although at the expense of increased computational complexity. We also observe, through numerical examples, the NMF loses CFAR due to the presence of additive white noise while the proposed GLRT retains the CFAR property for a wide range of shape parameter values.

\section{A. ML PARAMETER ESTIMATION FOR THE KNOWN NOISE POWER CASE}

For simplicity, we assume $\sigma^{2}=1$ as the observations can be properly normalized. We derive the ML estimates for the unknown parameters under the two hypotheses ( $s$ under $\mathbf{H}_{0}, s$ and $a$ under $\mathbf{H}_{1}$ ).

- Under $\mathbf{H}_{0}$, we have the likelihood function:

$$
L(s ; \mathbf{x}) \propto \frac{1}{\|\mathbf{M}\|} \exp \left[-\mathbf{x}^{H} \mathbf{M}^{-1} \mathbf{x}\right]
$$

Since $\boldsymbol{\Sigma}$ is assumed to be positive definite and Hermitian, $\boldsymbol{\Sigma}$ can be diagonalized by a unitary 
transformation (a.k.a., eigen decomposition)

$$
\boldsymbol{\Sigma}=\mathbf{U} \boldsymbol{\Lambda} \mathbf{U}^{H}
$$

where $\mathbf{U}$ is a unitary matrix and $\boldsymbol{\Lambda}$ is a diagonal matrix whose diagonal elements, say, $\lambda_{i}$ for $i=1, \cdots, N$, are real positive. Then

$$
\mathbf{M}=\mathbf{U}\left(\operatorname{diag}\left(s \lambda_{1}+1, \cdots, s \lambda_{N}+1\right)\right) \mathbf{U}^{H}
$$

We get

$$
\begin{aligned}
\|\mathbf{M}\| & =\prod_{i=1}^{N}\left(s \lambda_{i}+1\right) \\
\mathbf{M}^{-1} & =\mathbf{U}\left(\operatorname{diag}\left(\frac{1}{s \lambda_{1}+1}, \cdots, \frac{1}{s \lambda_{N}+1}\right)\right) \mathbf{U}^{H}
\end{aligned}
$$

Therefore

$$
\begin{aligned}
\frac{\partial\|\mathbf{M}\|}{\partial s} & =\sum_{i=1}^{N} \frac{\lambda_{i}\|\mathbf{M}\|}{s \lambda_{i}+1}=\operatorname{Tr}\left(\mathbf{\Sigma} \mathbf{M}^{-1}\right)\|\mathbf{M}\| \\
\frac{\partial \mathbf{M}^{-1}}{\partial s} & =\mathbf{U}\left(\operatorname{diag}\left(\frac{\lambda_{1}}{\left(s \lambda_{1}+1\right)^{2}}, \cdots, \frac{\lambda_{N}}{\left(s \lambda_{N}+1\right)^{2}}\right)\right) \mathbf{U}^{H} \\
& =\mathbf{\Sigma M}^{-2}
\end{aligned}
$$

where $\operatorname{Tr}(\mathbf{A})$ is the trace of matrix $\mathbf{A}$. By taking the derivative of $L(s ; \mathbf{x})$ with respect to $s$ and setting it to 0 , we get

$$
\operatorname{Tr}\left(\boldsymbol{\Sigma} \mathbf{M}^{-1}\right)=\mathbf{x}^{H} \mathbf{\Sigma} \mathbf{M}^{-2} \mathbf{x}
$$

Numerical procedure can be easily constructed to get the ML estimate for $s$.

- Under $\mathbf{H}_{1}$, the likelihood function is

$$
L(a, s ; \mathbf{x}) \propto \frac{1}{\|\mathbf{M}\|} \exp \left[-(\mathbf{x}-a \mathbf{v})^{H} \mathbf{M}^{-1}(\mathbf{x}-a \mathbf{v})\right]
$$

and by taking the derivative of $L(a, s ; \mathbf{x})$ with respect to $s$ and $a$ and setting them to 0 , we can get

$$
\begin{aligned}
\operatorname{Tr}\left(\boldsymbol{\Sigma} \mathbf{M}^{-1}\right) & =(\mathbf{x}-a \mathbf{v})^{H} \mathbf{\Sigma} \mathbf{M}^{-2}(\mathbf{x}-a \mathbf{v}) \\
a & =\frac{\mathbf{v}^{H} \mathbf{M}^{-1} \mathbf{x}}{\mathbf{v}^{H} \mathbf{M}^{-1} \mathbf{v}}
\end{aligned}
$$

Solving these two equations using numerical solution, we can get the ML estimates for $s$ and $a$.

\section{REFERENCES}

[1] E. Conte, M. Lops, and G. Ricci, "Asymptotically optimum radar detection in compoundGaussian clutter," IEEE Trans. Aerospace Electron. Systems, vol. 80, pp. 615-625, April 1995.
[2] F. Gini, "Sub-optimum coherent radar detection in a mixture of K-distributed and Gaussian clutter," IEE Proc. F, Radar, Sonar and Navigation, pp. 39-48, Feb. 1997.

[3] M. Rangaswamy and J.H. Michels, "A parametric multichannel detection algorithm for correlated non-Gaussian random processes," in Proc. IEEE National Radar Conference, Syracuse, NY, 1997.

[4] E. Conte, M. Lops, and G. Ricci, "Adaptive detection schemes in compound-Gaussian clutter," IEEE Trans. Aerospace Electron. Systems, vol. 34, pp. 1058-1069, Oct 1998.

[5] K.R. Gerlach, "Spatially distributed target detection in non-Gaussian clutter," IEEE Transactions on Aerospace and Electronic Systems, vol. 35, pp. 1058-1069, 1999.

[6] F. Gini, M.V. Greco, and A. Farina, "Clairvoyant and adaptive signal detection in nonGaussian clutter: A data dependent threshold interpretation," IEEE Trans. Signal Processing, pp. 1522-1531, 1999.

[7] E. Conte and M. Longo, "Characterisation of radar clutter as a spherically invariant random process," IEE Proceedings, part F: Radar, Sonar, and Navigation, vol. 134, pp. 191-197, April 1987.

[8] M. Rangaswamy, D. Weiner, and A. Ozturk, "Non-Gaussian random vector identification using spherically invariant random processes," IEEE Trans. Aerospace and Electronic Systems, vol. 29, pp. 111-123, Jan 1983.

[9] L.L. Scharf and T.L. McWhorter, "Adaptive matched subspace detector and adaptive coherence estimators," in Proc. 30th Asilomar Conference on Signals, Systems, and Computers, Pacific Grove, CA, Nov. 1996.

[10] L.L. Scharf, T.L. McWhorter, and L.J. Griffiths, "Adaptive coherence estimation for radar signal processing," in Proc. 30th Asilomar Conference on Signals, Systems, and Computers, Pacific Grove, CA, Nov. 1996.

[11] S. Kraut, T.L. McWhorter, and L.L. Scharf, "A canonical representation for the distributions of adaptive matched subspace detectors," in Proc. 31st Asilomar Conference on Signals, Systems, and Computers, Pacific Grove, CA, Nov. 1997. 
[12] S. Kraut and L.L. Scharf, "The CFAR adaptive subspace detector is a scale-invariant GLRT," IEEE Trans on Signal Processing, vol. 47, pp. 2538-2541, Sept 1999.

[13] J.H. Michels, M. Rangaswamy, and B. Himed, "Performance of STAP tests in compoundGaussian clutter," in Proc. IEEE Sensor, Array and Multichannel Signal Processing Workshop, Cambridge,MA, March 2000.

[14] J.H. Michels, B. Himed, and M. Rangaswamy, "Performance of STAP tests in Gaussian and compound-Gaussian clutter," Digital Signal Processing, vol. 10, pp. 309-324, Oct. 2000.

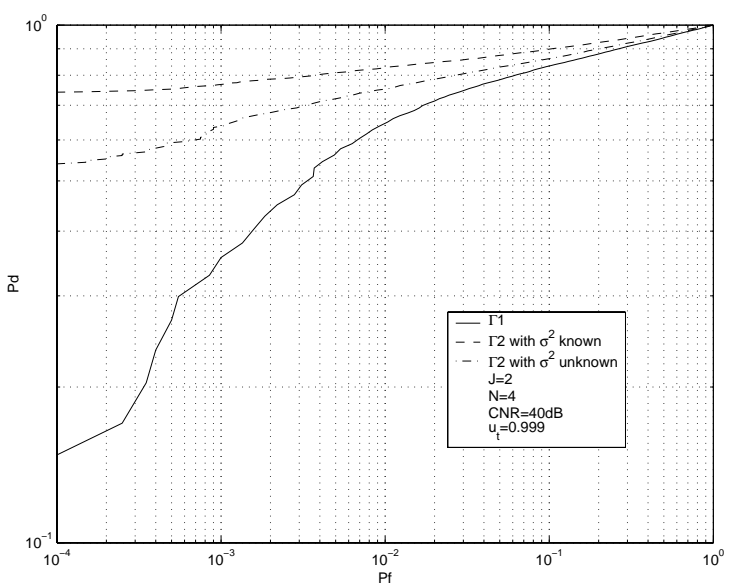

Fig. 1: Performance comparison between the two GLRT $\left(\Gamma_{1}\right.$ and $\left.\Gamma_{2}\right)$ in the presence of $\mathrm{K}$ distributed clutter and additive white Gaussian noise.

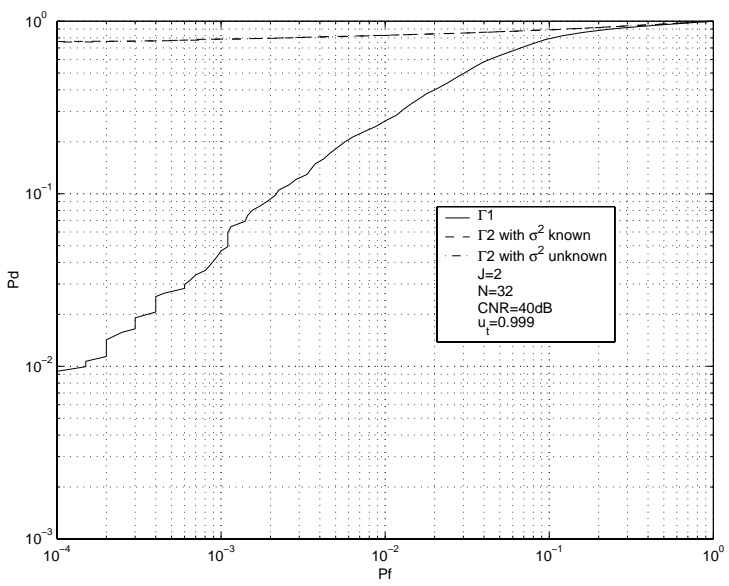

Fig. 2: Same as in Fig. 1 except that $N=32$ instead of 8 .

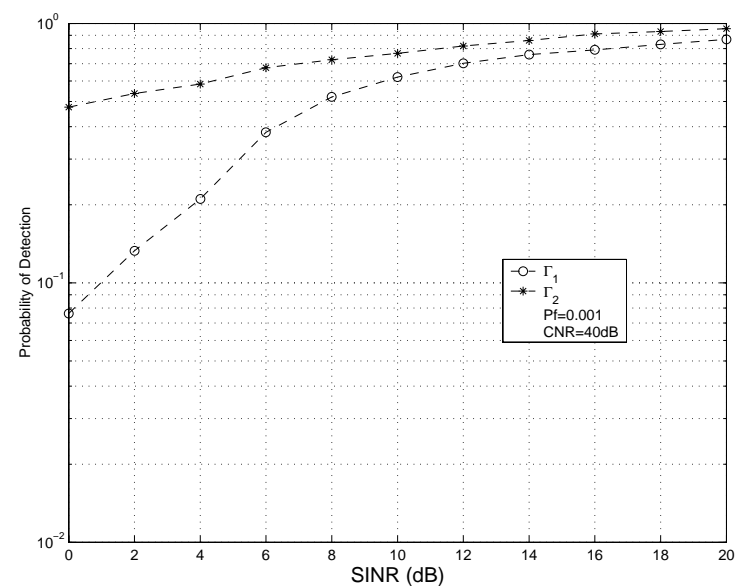

Fig. 3: Probability of Detection as a function of SINR.

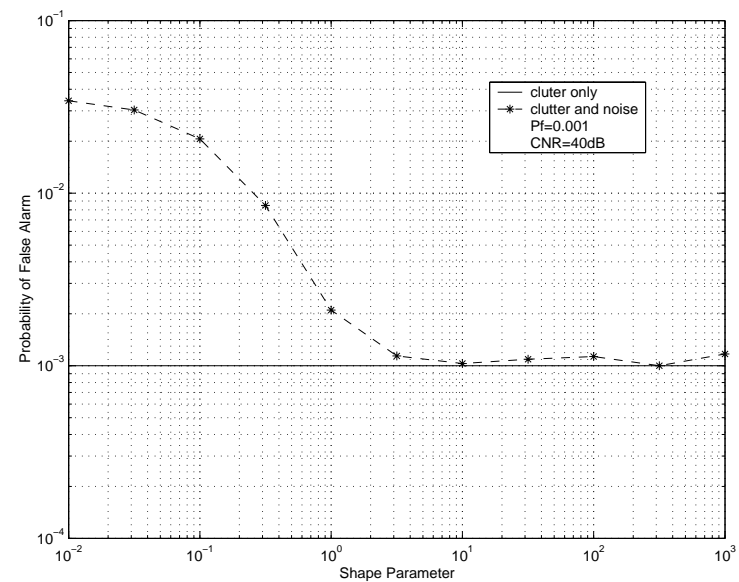

Fig. 4: Flase alarm rate as a function of the shape parameter in the $\mathrm{K}$ clutter and additive white Gaussian noise for $\Gamma_{1}$.

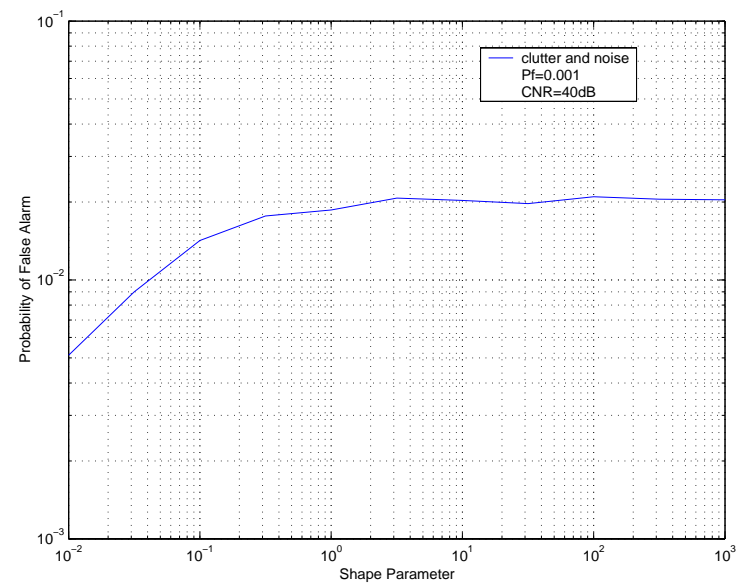

Fig. 5: Flase alarm rate as a function of the shape parameter in the $\mathrm{K}$ clutter and additive white Gaussian noise for $\Gamma_{2}$. 